\title{
UN ESTUDIO DE LA REPRESENTACIÓN SOCIAL «DE LA LEY» ENTRE LOS ADOLESCENTES
}

\section{A STUDY OF THE SOCIAL REPRESENTATION OF «THE LAW» AMONG TEENAGERS}

\section{UNE ÉTUDE DE LA REPRÉSENTATION SOCIALE « DU DROIT » CHEZ LES ADOLECENTS}

\author{
Johanne Masclet \\ Universidad Lille III, Francia
}

\begin{abstract}
Resumen: El actual contexto social nos lleva a plantear la temática de la relación existente entre los adolescentes y la ley. A comienzos del siglo pasado, Piaget intentó comprenderla por medio del estudio del juicio moral en el niño. Desde entonces, los trabajos sobre el tema no han cesado de evolucionar. Para este estudio relacionamos Ley/Derecho y Psicología y hemos estudiado la representación social de la Ley en tres poblaciones: alumnos de secundaria, profesores de secundaria y abogados.

Presentamos la hipótesis de que, dependiendo del contexto y experiencia social, las representaciones sociales de la Ley de las diferentes muestras, contribuirán a la diferenciación entre éstas últimas, así como entre diferentes niveles de contenido o estructura. La muestra empleada para este estudio está compuesta por 338 sujetos distribuidos de la siguiente manera: 196 estudiantes de secundaria de 3er año, 78 profesores de secundaria, 64 abogados. Desde el punto de vista metodológico, optamos por emplear la teoría del núcleo central de la escuela Aixois, adaptada para nuestra investigación.

Se empleó un método de asociación de palabras, conteniendo la frase "la Ley" para la recogida de datos. El método fila/frecuencia (Cañas, 1972; Abric, 2003) fue el seleccionado para la implementación. Por último, utilizando el índice de Jaccard, intentamos comprender la estructura de las representaciones sociales.
\end{abstract}

Palabras clave: Derecho, adolescentes, representación social.

\begin{abstract}
The current social context brings arround the subject of the relationship between teenagers and the Law. At the beginning of the last century, Piaget already sought to understand it through the study of the moral judgment of the child. Since then, work on the subject did not cease evolving. For this study we connected Law and Psychology and we studied the social representation of Law on three different populations: secondary school pupils, high school teachers and lawyers. We put forward the assumption that, depending on their context and social experience, sample's social representations of Law shall contribute to distinguishing between them as well as between different levels of contents or structure. The sample of this study is composed of 338 subjects distributed as follows: 196 high school students of 3rd year, 78 high school teachers, 64 lawyers. From the methodological point of view, we chose to use the Aixoise School's theory of central core, adapted for our research.

A word association method, containing the phrase "the Law" was used in order to collect our data. Subsequently, the row/frequency method (Rods, 1972; Abric, 2003) imposed itself for the implementation. Finally, using the Jaccard index, we attempted to understand the structure of the social representations.
\end{abstract}

Keywords: Law, teenager, social representation.

\section{INTRODUCCIÓN}

El contexto social actual nos ha llevado a planteamos la cuestión de la relación que mantienen los adolescentes con el derecho (se mencionará indistintamente derecho o ley, salvo cuando se los defina explícitamente, $\mathrm{N}$. E.). En efecto, las cuestiones de violencia, de incivilidad, de no respeto del otro parecen ser una preocupación del entorno educativo. Es en este marco donde hemos llevado una investigación entre adolescentes de 14 años.

La cuestión de la relación con la regla y la ley la plantearon ya numerosos autores. En 1932, Piaget intentaba entender el desarrollo del juicio moral en el niño. 
Desde entonces los estudios sobre el tema no han dejado de desarrollarse: Kohlberg (1971), Kourilsky (1991-2002), Tostain (1999), Lehalle, H., Aris, C., Buelga, S y Musitu, G. (2004). Gangloff y Hardy-Massard (2006) han establecido así una relación directa entre derecho y psicología. Estudiaron más particularmente la noción de sanción y sus criterios de atribución. Para el presente estudio pusimos también en relación derecho y psicología, pero hemos enfocado el problema de otra manera, utilizando el concepto de Representacion Social. Bien se sabe que dicho concepto abarca tanto las creencias como los conocimientos, las opiniones, las actitudes producidas y compartidas por individuos de un mismo grupo ante un objeto social dado. Pensamos que en tomo al objeto social «derecho», podrían existir desafíos y hasta conflictos de representaciones entre adolescentes y adultos. Pensamos que esta diferencia puede ser el origen de ciertos pasos a la acción de adolescente. Las especulaciones teóricas relativas al derecho nos lo enseñan pero estamos dispuestos a demostrarlo mediante un enfoque experimental. Esperamos recoger bastantes datos para dar respuesta a los problemas inicialmente planteados.

\section{MARCO TEÓRICO DEL ESTUDIO}

\section{EL MARCO JURÍDICO}

Para Planiol (1934) «La palabra derecho abarca el conjunto de leyes, es decir reglas juridicas aplicables a los actos de los hombres» (p.l). Según Carbonnier (1911), «el derecho es el conjunto de las reglas de derecho, tal y coma se ve expresado en las fuentes formales, ley y costumbres ... » (p.3). No se puede pues definir como conjunto de las reglas de conducta que en la sociedad rigen las relaciones de los hombres entre sí. Además la especificidad de estas reglas es que ellas se imponen a ellos, incluso mediante el imperativo estatal. De hecho, se diferencian de otras reglas de conducta que estructuran la vida en la sociedad, particularmente las reglas de urbanidad, religiosa o también moral. Pues si la regla de derecho se impone al hombre, parece necesario interrogarse sobre su definición y su fundamento.

Su definición suscita controversia. A mediados del siglo pasado, el Pr Dabin (1969) comprobó cuan dificil resultaba dar de ella una defnición satisfactoria, tan inagotables parecían ser los debates ideológicos sobre el tema.
Asi por ejemplo Buffelan-Lanore (2007) explica que la regla de derecho responde a dos necesidades: necesidad de seguridad y necesidad de justicia. En cuanto a la primera necesidad, ella permite diferenciar lo que podemos hacer de lo que hemos de tolerar por parte de los demás. En cuanto a la segunda, la idea es que el hombre tolera que una regla de derecho limite sus deseos a condición de que ésta sea justa. Entonces dos preguntas surgen, ¿puede dicha necesidad de justicia verse cumplida por la regla de derecho y puede basarse el derecho en la justicia?

Los debates sobre el tema son complejos pero podemos dar unas pistas de reflexiones que podrían aclararlos: Para contestar a estas dos preguntas, dos doctrinas suelen oponerse: la doctrina idealista o escuela del derecho natural y la doctrina positivista. Según la escuela de derecho natural, la regla de derecho tiene su fundamento en la justicia. Esta corriente de pensamiento es muy antigua y aparece ya en la filosofia griega. Así cuando el Rey Creón le reprocha a Antígona haber enterrado a su hermano a pesar de que se le hubiera prohibido terminantemente, Sófocles le mandó contestar: «No pensaba yo, dijo Antígona, que sus órdenes pudiesen prevalecer frente a la voluntad de los inmortales, frente a estas leyes que no están escritas y que no podrían ser borradas. No es nada nuevo la existencia de estas leyes: son de todas las épocas y nadie puede decir cuándo nacieron».

Esta teoría va a desarrollarse con el cristianismo y los antiguos autores franceses. Podemos citar en particular a Santo Tomás de Aquino quien difunde y profundiza el pensamiento de Aristóteles y distingue tres leyes: la lex aeterna (o ley divina); la lex naturalis (ley de la razón: lo que conoce el hombre de la ley divina gracias a su razón); la lex humana que es el derecho positivo. El principio de cualquier ley humana ha de contribuir al bien común.

Esta teoria va a laicizarse en los siglos XVII y XVIII, en particular con el holandés Grotius y más tarde con J.J. Rousseau. En el Contrato social, éste explica que los hombres vivieron en un primer tiempo en el estado de naturaleza, y luego por el «contrato social» han abandonado al poder social una parte de su prerrogativa, pero sólo una parte. Han guardado ciertas latitudes, inherentes a la naturaleza humana tales como el respeto de la vida, la libertad de ir y venir, de conciencia, etc. 
Estos derechos son derechos naturales y el poder no puede atentar contra ellos. Al origen de este "contrato social», está la idea de que los gobernantes sólo actuan según los poderes que les otargó el pueblo.

La Declaración de los derechos del hombre y del ciudadano de 1789 por cierto adopta estos derechos naturales. El artículo 6 de la Declaración de los derechos del hombre y del ciudadano precisa que la ley es «la expresión de la voluntad general».

Esta teoría del derecho natural fue criticada en los siglos XVII y XVIII pero sobre todo en el siglo XIX bajo la, influencia del jurisconsulto alemán Savigny así como de la Escuela histórica. Según ellos, el derecho no es más que el resultado de la evolución histórica de un país. El ideal que concebimos se ve sometido a la evolución. Lo que antaño parecía injusto puede parecer justo hoy dia. No significa esto que el Ideal de justicia no existe sino que la concepción que tenemos de éste evoluciona.

La crítica esencial resulta de la dificultad de estar de acuerdo sobre el contenido del derecho natural. Además, los que tratan de definirlo necesariamente se ven influenciados por sus opiniones filosóficas, sus creencias religiosas. Pascal decía «Graciosa justicia a la cual limitan un río o una montaña! Verdad aqui es error tras los Pirineos». Como lo recuerda BuffelanLanore (2007), es verdad que ciertos principios que parecían fundamentales como el respeto de la vida humana, no siempre lo han sido, asi el pater familias romano tenía derecho de vida y de muerte sobre sus hijos. Era lo mismo a propósito de la esclavitud. Ahora bien, esta variabilidad del derecho natural está en oposición con su definición como derecho universal e inmutable. En realidad cuando en la práctica se lleva al paroxismo la teoria del Derecho Natural, que justifica la resistencia frente a cualquier ley injusta, ésta puede llevar a la anarquia.

Opuesta a esta visión está la concepción positivista la que no reconoce otro derecho sino el derecho positivo, es decir el conjunto de las reglas de derecho objetivas vigentes en un Estado en un momento dado. Lo que cuenta es la eficacia: la ley ha de ser respetada, no porque es justa, sino porque es la regla la que garantiza la seguridad, el bien común, de ser necesario por la fuerza. Este derecho no se justifica por el recurso a un ideal, en particular el Ideal de justicia, se impone por el mero hecho de que lo decrete un soberano.
Este positivismo se manifiesta esencialmente por tres corrientes de pensamiento: el positivismo estatalista o juridico, el positivismo sociológico, el positivismo marxista.

La primera corriente de pensamiento considera al Estado como única fuente de derecho positivo, que es el único derecho. Courbe (2003) explica que en el signlo XIX, para Ihering, jurista alemán, el derecho es el fruto de un combate permanente, es «la política de la fuerza». En una sociedad, los imperativos del Estado priman sobre todos los demás. Es única fuente del derecho. Courbe (2003) recuerda igualmente que en el siglo XX, según Kelsen, las normas jurídicas se organizan en una pirámide. Cada norma saca su fuerza obligatoria de su conformidad con la norma superior. El Estado es la única entidad que designa esta planificación jurídica y la concepción del derecho tiene que estar depurada de cualquier clase de ideologia.

Para los positivistas sociológicos, se plantea la cuestión del derecho de otro modo. El francès Auguste Comte es uno de los fundadores de esta Escuela y Durkheim adoptó sus ideas después. Para ellos, el fundamento de la regla de derecho ya no se busca en la voluntad del Estado sino en la conciencia de las masas. El derecho dentro de esta concepción es un hecho social, es el reflejo de la observación de una realidad social, no emana de la voluntad discrecional de los gobemantes, sino que lo impone la conciencia colectiva del grupo. En efecto, en la práctica, resulta dificil imponer una regla de derecho que no sea admitida por el cuerpo social y el legislador se ve a veces llevado a modificar una regla vigente para volverla de conformidad con los deseos del cuerpo social.

El derecho entonces es un producto de la evolución del pueblo y la ley tan sólo consagra el resultado de esta creación histórica. Por fin, para la última corriente de pensamiento, resultante de la ideologia Marxista, el derecho es un producto de la economia. Courbe (2003) explica que para Marx el derecho positivo expresa los intereses de la clase dominante, la que es propietaria de los medios de producción. La idea es que la evolución de las relaciones de producción (factores económicos) es la que constituye la infraestructura de la sociedad, y la que determina las transformaciones de la superestructura (las ideologías) y por lo tanto, el derecho. Entonces si la lucha de clases se acaba por la liberación del proletariado, las clases desaparecen; ya no existe pues imperativo ni desestimación del derecho en la sociedad comunista ideal. 
Estas doctrinas positivistas por cierto provocaron varias críticas y acarrearon ciertas preguntas. Para unos, ¿puede la sola evolución social justificar que un Estado imponga tal regla y no otra? Por otra parte, admitir la concepción del derecho tal y como la enfocan los positivistas etáticos, lleva a decir que tendríamos que aceptar entonces las reglas más despóticas sin preguntarnos si son justas o no. Encontraría así el totalitarismo toda su justificación.

Frente a las corrientes de pensamiento que todo parece oponer, la primera con tendencia individualista, en que la sociedad no tiene existencia propia y en la cual el objetivo buscado es el respeto de la actividad de cada individuo; la otra más socialista, en la cual sólo la sociedad tiene valor permanente y donde el objetivo buscado es el interés social; ¿Qué actitud adoptar?

Para Buffdan-Lanore (2007) hay que buscar de hecho un equilibrio entre el interés general y los intereses individuales para garantizar los objetivos económicos, sociales políticos deseados por el Estado, o sea el bien común.

En el siglo XVIII, Portalis decía del derecho natural que había de ser como una brújula para el derecho positivo. En cierto modo, decía él que el derecho natural sería un valor moral, un elemento de comparación que permitiera interpretar las leyes y guiaría al legislador.

Esta breve exposición permite constatar que el derecho es de verdad un objeto complejo. El equilibrio entre estos dos puntos de vista puede resultar difícil en la práctica, cuando se trata de posicionarse en uno de los dos platillos de la balanza, símbolo de justicia.

Este objeto de estudio no lo han olvidado las ciencias humanas. En efecto si el derecho forma parte de nuestra organización social, los individuos tendrán de él inevitablemente una percepción, una representación. Es lo que ciertos investigadores en psicología han tratado de estudiar.

\section{EL MARCO PSICOLÓGICO}

\section{ELENFOQUECOGNITODELASOCIALZACIÓN JURÍDICA}

Para Piaget (1932), la idea de justicia es en primer lugar una cuestín de moral. Según él, existe un paralelismo entre desarrollo moral y desarrollo intelectual del niño. Sus estudios han puesto de realce una relación entre la naturaleza de las reglas de juego de los niños y su juicio moral.
Lo llevaron a la conclusión de que podrían existir dos fases en el desarrollo moral del individuo. La primera es la heteronomía, en la cual obedece el niño a las reglas del adulto sin conciencia de la necesidad social. La obediencia a la regla se basa en el respeto unilateral del niño para con los mayores. En la segunda fase, el niño va tomando conciencia de los principios de reciprocidad y de igualdad. La obediencia se basa entonces en el respeto mutuo entre individuos considerados como iguales. PagoniAndreani (1999) explica que los fundamentos de esta autonomía son esencialmente racionales y ésta se apoya en un sistema formal de relaciones interpersonales construido en la interacción de la persona con su entorno. Esto lleva a una concepción revolucionaria de la educación moral, tradicionalmente basada en la aceptación pasiva de las reglas sociales ya vigentes. Tapp y Kohlberg (1971) al inspirarse en estos trabajos, van a descubrir los estados de la conciencia moral y jurídica. Pagoni-Andréani (1990) explica que, según ellos, existen tres niveles de desarrollo, cada uno de éstos dividido en dos fases.

Al primer nivel lo suelen llamar «pre-convencional». La fase 1 , se ve marcada por el egocentrismo del individuo quien admite con dificultad un punto de vista diferente del suyo. Una acción es justa si está de conformidad con la regla. En la fase 2, la perspectiva es individualista, con la posibilidad de reconocer el punto de vista ajeno. La acción justa es la que respeta las reglas, y que además está en conformidad con sus propios intereses.

Al segundo nivel lo suelen llamar "convencional». En la fase 3, el niño tiene la idea de que lo que hay que hacer no sólo está relacionado con su propio interés o con una autoridad cualquiera. Una acción justa es la que está en adecuación con los papeles atribuidos por el entomo. En la fase 4, el individuo se interroga sobre el objetivo y el papel social de las leyes. La acción justa es la que sostiene el orden social y el individuo sigue su conciencia social para cumplir su deber con respecto a la sociedad.

Al tercer nivel lo suelen llamar «post-convencional». En la fase 5, el individuo se adhiere libremente al principio de las sociedades democráticas. Tostain (1999) habla de «crisis del relativismo» (p. 108). La persona, frente a otros grupos o sociedades, toma conciencia de que existen normas y sistemas de valores distintos de los vigentes en su propia sociedad. 
Aqui la acción justa es la que defiende las reglas sociales establecidas, pero el individuo tiene conciencia de que cada grupo social tiene sus propios valores.

Por fin en la fase 6, según Tostain (1999), las personas consideran las leyes como válidas cuando se apoyan en el respeto de principios éticos universales. Estos giran en tome a ideas más generales de justicia tales como la vida, la libertad, la igualdad de los derechos de los hombres.

Para Kohlberg (1971), no todos los individuos alcanzarán necesariamente la fase 6 . Además, según él, para acceder a una fase, el paso por las fases inferiores es inevitable.

Los modelos que se han inspirado en estas investigaciones han sido criticados, en particular por el hecho de que consideren dicho modelo como universal. Tostain (1999) explica que varios estudios (Sampson, 1977; Simmson, 1974; Shcweder, 1982; Sullivan, 1977) afirman que el modelo de Kohlberg podría conllevar una perspectiva etnocéntrica pro-occidental. En este marco Snarey (1985), al desear quedarse en el plano puramente cognitivo, propone una síntesis de de los trabajos de Kohlberg a propósito de la invariancia de la secuencia de adquisicion de las fases, a partir del estudio de 27 áreas culturales distintas. Muestra que en el $85 \%$ de los casos la adquisición se hace en el orden previsto por Kholberg. Concluye que las fases 1 a 4 pueden ser consideradas como universales pero que la presencia de las fases 5 y 6 es escasa.

Según Lehalle y al. ( 2004 ) se han utilizando los procedimientos de Kholberg en el Moral Judgment Interview ( MTI ) (Colby \& Kholberg, 1987; Colby, Kholberg, Speicher, Hewer, Candee, Gibbs \& Power, 1987). 0 sea, utilizando el cuestonario - test de Rest le DIT- Defining Issues Test - (Rest, 1979 ; Rest, Davison y Robbins, 1978; Rest, Narvaez, Bebeau \& Thomas, 1999) los estudios demostraron efectivamente la débil frecuencia de las fases 5 y 6 dentro de la población general ( adolescentes y adultos ).

Según Lehalle y al. (2004), sin embargo, desde un punto de vista estrictamente desarrollista, la descripción de una evolución no puede ser tachada de inválida con el pretexto de que las fases últimas de un desarrollo suelen ser poco frecuentes. En efecto, un sistema de fases indica un modo de abrirse camino necesario, si la persistencia de los ruegos medioambientales adecuados lo hace posible.
No se trata pues en absoluto de una evolución predeterminada que tenga que producirse a edades bien identificadas. Lehalle y al. (2004) piensan que el nivel de educación podria influir en su emergencia.

\section{LA INFLUENCIA DEL CONTEXTO SOCIAL EN LA SOCIALIZACIÓN JURÍDICA}

Percheron (1991) con respecto a los trabajos de Piaget explica que el desarrollo que describe no puede considerarse como dependiente sólo del mundo cognitivo. Además, ella precisa que las personas estudiadas por éste se hallaban en la niñez o al principio de la adolescencia. Ahora bien, la socialización no siempre se detiene con la adolescencia. Apoyándose en los datos de una encuesta de opinión de 1987, entre franceses de 16 a 21 años, ella muestra la evolución de la concepción de las leyes entre estas personas. Ella señala la acción de diversas variables en el fenómeno de socialización: el sexo, la pertenencia sociocultural, la experiencia, etc. Deduce de ello que la identidad social y las experiencias de la vida de la persona podrían influir en sus percepciones de la ley y de la justicia, y por consiguiente en sus actitudes. Kourilsky (1990) se interesó por el fenómeno de socialización jurídica. Lo define como el proceso mediante el cual la persona asimila los principios fundamentales del Derecho que rige su sociedad, en su sistema de representaciones y de conocimientos. Mediante este proceso la persona haria suya su identidad jurídica, su identidad como persona del Derecho y persona de derechos. Esta apropiación se produce en dos etapas. En un primer lugar, la persona interiorizaría las representaciones dominantes del Derecho en la cultura de su sociedad. Luego habría una especie de aculturación por la persona de los conceptos juridicos. Dicho de otro modo la persona volvería a crear dichos conceptos en función de sus propios valores con el propósito de darles un sentido en su propia cultura.

En resumen, la persona juega entre la cultura jurídica dominante y su propia cultura. La primera concieme las normas y los saberes colectivos, relativos al derecho de un país. La segunda se refiere a sus propias representaciones, construidas a partir de saberes y valores transmitidos por el grupo al cual pertenezca. Así, muy pronto, el niño aprende lo que tiene el derecho de hacer o no, lo que ha de hacer, como niño. 
Después, sus aportaciones sociales se enriquecen y se vuelven más complejas y le permiten ir entendiendo durante conversaciones o cursos cuáles son los derechos, deberes, la relación con el derecho, en la sociedad en la cual vive. Va construyéndose pues a medida que las recibe y en función de las lecciones adquiridas en su entorno familiar y escolar así como por sus experiencias personales, un sistema de valores y de representaciones personales, entre las cuales figuran en particular las que están relacionadas con el derecho.

En otra investigación realizada en Francia y Polonia entre poblaciones similares de 11-12 años, de 13-14 años y de 16-18 años, relativas a la evolución desarrollista según la edad de la aculturación juridica, Kourilsky (1991) señala una diferencia de concepción del derecho entre los jóvenes de 13-14 años franceses y polacos. En efecto, en Francia, los adolescentes asocian en un primer tiempo el derecho a «la libertad», y después al «permiso». En Polonia, por el contrario, el derecho está más asociado a «la autoridad».

Por otra parte, aunque en las dos culturas la ley está mayormente representada, entre los 11-12 años y los 13-14 años, como algo imperativo y que se impone a todos, mediante obligaciones y prohibiciones, esta representación parece ser más fuerte entre los $16-18$ polacos cuando se modifica entre los franceses. En éstos, la noción de norma aceptada se impone sobre la noción de ley imperativa.

Esta desemejanza podría explicarse, según Kourilsky (1991) por la diferencia de régimen político, ya que conoció Polonia durante todo un periodo un sistema político autoritario.

En un estudio longitudinal llevado desde 1993 hasta 2000 Kourilsky (2002) muestra por otra parte que el cambio de contexto social ha sido una variable fundamental en la representación del derecho en el ciudadano ruso. La representación penalista y represiva del derecho, predominante observada en éste en el 93 y que se podia comparar con la fase preconvencional de Kohlberg y de la fase de la heteronomia de Piaget va difuminándose y en el 2000 la reemplaza una representación más parecida a la de los franceses en el 93.

En efecto, Kourilsky (2002) señala un desarrollo a lo largo de la adolescencia que va hacia una concepción "socializada» y «juridicalizada» de la igualdad (igualdad ante la ley, igualdad de los derechos), de una libertad vista con el enfoque de las libertades cívicas (libertad de opinión, libertad de pensamiento) y sobre todo de la aparición de reflexiones sobre la necesidad de los límites aportados a la libertad de uno por la libertad de otro. El ciudadano enuncia y reivindica sus derechos y libertades.

El interés de tales estudios es mostrar que la aprehensión del derecho puede evolucionar. Si Piaget y Kholberg demuestran que esta evolución no se hace por casualidad y que podrían existir fases de desarrollo concomitante al desarrollo intelectual, otros señalan que dicho desarrollo se ve estimulado por variables del entomo, tales como el contexto educativo o también el contexto social del individuo. En nuestra opinión, estos contextos no son independientes unos de otros, en la medida que el contexto educativo institucional de un país suele depender del contexto social y político del propio país.

Este análisis teórico lleva a decir que si tuviéramos que volver a situar el enfoque positivista y el enfoque idealista del desarrollo de la socialización jurídica, diríamos que el primero está más cerca de la fase convencional de Kholberg y el segundo de su fase postconvencional.

Fue con esta idea con la que hemos emprendido el estudio de la representación del derecho de tres poblaciones distintas: es decir una población de adolescentes, una población de docentes, una población de juristas.

\section{MÉTODO}

En función de lo que está expuesto mas arriba, tomamos como hipótesis que la representación social de adolescentes de tercero de BUP se diferencia de la de los docentes del colegio y la de los juristas. La población de los juristas está compuesta esencialmente de profesores no-titulares de la universidad que tienen una actividad de juristas en terrenos próximos a los colegios escogidos. Esta elección ha permitido una movilización más fácil de estos profesionales. Nuestro trabajo consistirá también en poner de realce los campos de esta diversidad y en volver a situarla en nuestro contexto teórico. 


\section{PARTICIPANTES}

La muestra se compone de 338 personas dividas de la manera siguiente (ver cuadro 1) entiende como conjunto de objetos sociales que tienen entre sí relaciones de jerarquía y de oposición, que estructuran «la repartición de un capital específico de valor social».

\begin{tabular}{lccc}
\hline Poblaciones & Hombres & Mujeres & Totales \\
\cline { 2 - 4 } Alumnos & 102 & 94 & 196 \\
\cline { 2 - 4 } Docentes & 37 & 41 & 78 \\
\cline { 2 - 4 } Juristas & 29 & 35 & 64 \\
\hline & \multicolumn{2}{c}{ Cuadro 1- Distribución de las poblaciones }
\end{tabular}

\section{INSTRUMENTOS}

\section{ELECCIÓN TEÓRICA DE LOS ELEMENTOS UTLZADOS PARA LA INVESTIGACIÓN : LA NOCIÓN DE « REPRESENTACIÓN SOCIAL»}

La representación es «el producto y el proceso de una actividad mental mediante la cual individuos vuelven a construir la realidad y le atribuyen una significación especifíca » (Abric, 1976).

El pensamiento social está centrado en la comunicación, en la noción de interracción. Si un objeto constituye un desafío social (aunque que sea limitado), la representación correspondiente será el producto de un amplio conjunto de interracciones (internas en el mismo grupo). La representación social está generada pues de manera colectiva, y por lo tanto está compartida.

En cuanto a la cuestión de la estructuración de las representaciones sociales, existen dos grandes campos teóricos que no son en absoluto antagonistas: la escuela de Ginebra con su teoría de los principios generadores de postura y la escuela de Aix con su teoría del núcleo central.

La presentación de estos dos campos nos permite argumentar nuestra elección preferente de la teoría del núcleo central, más adaptada a nuestra investigación.

\section{LA TEORÍA DE LOS PRINCIPIOS GENERADORES DE POSTURAS}

Para Doise y Palmonari (2002) las representaciones sociales son «principios generadores de posturas vinculadas a intenciones específicas en un conjunto de relaciones sociales y organizan los procesos simbólicos que intervienen en dichas relaciones» (p. 124). Este doble origen nos lleva a evocar una noción clave para Bourdieu, la noción de campo, campo que se
La gran diferencia entre la teoría del núcleo central (Abric, 1976) y la teoria de los principios generadores de postura (Doise ,1986) se sitúa en la noción de estructura. Para Doise, la estructura de la representación es una estructura de datos (análisis factorial), o sea las relaciones entre elementos de un campo representacional. Esto se manifiesta por:

- relaciones de interdependencia o de analogía

- relaciones de independencia o antagonismo.

El interés de esta teoría se basa en el hecho de que ponga un vínculo directo entre la representación social y la postura, es decir de hecho la actitud de un individuo ante un objeto social dado. La actitud es, según Moscovici «la dimensión primera de la representación». Es decir el mediador entre la representación y las prácticas sociales de un individuo o de un grupo.

\section{LA TEORÍA DEL NÚCLEO CENTRAL}

La teoría del núcleo central (Abric, 1976) enriquece el cuadro teórico de Moscovici y vuelve a utilizar la idea del núcleo figurativo. Vamos a considerar que la representación funciona como una entidad (Abric 1976) que está organizada alrededor de un núcleo central cuya función es estructurante. Los elementos que componen este núcleo tienen entre sí relaciones con fuerte conexidad y constituyen por lo tanto un núcleo de significaciones preponderantes.

El núcleo central permite pues la gestión del sentido y proporciona los medios de su coherencia. Nunerosas experiencias muestran por otra parte que el replanteamiento de los elementos centrales genera un desplome del sentido en el individuo.

Sin embargo, la representación social funciona gracias a dos componentes distintos, pero complementarios: el sistema central y el sistema periférico. 
El sistema central es el fundamento de la estructuración de la representación. Por esta razón va a desempeñar un papel especifíco y fundamental, en particular por principio de economía, para la gestión de la representación.

En cuanto al sistema periférico está bajo la dependencia del sistema central. Los elementos que lo componen están ponderados y su valor lo determina en gran parte el sistema central.

Los elementos de conocimiento de ambos sistemas se llaman creencias centrales y creencias periféricas.

Muchísimas experiencias y publicaciones tratan de la oposición entre creencias centrales y creencias periféricas (Abric 1994). Podemos resumir la función de las dos dimensiones de la representación de la manera siguiente:

El sistema central es:

- estable, coherente y rígido

- vinculado a la historia del grupo

- vinculado a la memoria colectiva

- «el lugar de consenso de las representaciones sociales», define la homogeneidad del grupo resistente al cambio

- garante de la perennidad de la representación

- poco sensible, hasta insensible al contexto inmediato

El sistema periférico es:

- flexible y suave, soporta la contradicción

- permite la integración de las experiencias y de las historias individuales

- soporta la heterogeneidad y la diversidad en el sena del grupo

- evolutivo, adaptativo

- sensible al contexto inmediato

Es importante constatar que el sistema periférico desempeña el papel de «tampón» que permite conservar la estructura del sistema central. Además, el hecho de que sea evolutivo permite defender la representación, según el contexto, y concretar el sistema central (cuyas creencias han de seguir consensuales y estructuradas para que no se pierda el sentido de la representación). El sistema periférico juega también un papel de mediación con el entorno; gracias a los elementos periféricos es coma la representación se arraiga en la realidad.

\section{JUSTIFCACIÓN DELA日ECCIÓN TEÓRICA DE AIX}

Como tuvimos ya la oportunidad de justificarlo en la exposición teórica, no buscamos por qué la representación social del derecho justificaría la postura de los adolescentes a propósito de su objeto, sino cómo ella lo hace legítimo, es decir la actitud de un individuo ante este objeto social.

\section{PROCEDIMIENTO}

\section{EL NÚCLEO CENTRAL DE LA REPRESENTACIÓN}

Existen tres diferentes técnicas que permiten identificar el núcleo central de una representación:

- el método clasificación / frecuencia (Verges, 1992)

- el método de los esquemas cognitivos de base (Guimelli \& Rouquette, 1992)

- la inducción por guión ambiguo (Moliner, 1993)

- el análisis de similitud (Falment, 1962)

- la técnica de planteamiento (Moliner, 1988).

Por motivos de comodidad y de eficacia, un método de asociación de palabras, con el término«el derecho» ha sido utilizado para la recopilación de nuestros datos. Se utiliza este método en numerosos estudios sobre las representaciones sociales. Entonces fue el método clasificación/frecuencia el que se impuso con más evidencia para llevar a cabo el análisis de los resultados.

Según Verges (1992), es el puesto de aparición de las palabras asociadas el que hay que tomar en cuenta para la construcción experimental de la representación. Pero la técnica del puesto de importancia, según estudios más recientes, parece adecuarse mejor en la hipótesis de la existencia de una zona muda de las representaciones sociales (Abric,2003).

Con el objetivo de adoptar esta tesis se les pidió a las personas interrogadas un trabajo de tipo metacognativo sobre sus respuestas. Les invitamos a jerarquizar sus respuestas de 1 a 10 , del término más próximo al término menos próximo de la palabra derecho (1 correspondiendo al término más próximo y 10 correspondiendo al término más alejado).

Así, fueron seleccionados primero los elementos del campo representacional por un criterio preconizado en la mayor parte de los estudios (es decir los elementos cuya frecuencia $\mathrm{N}>10$ : elementos citados por más del $10 \%$ de las personas). 
Después, según el método clasificación/ frecuencia, los elementos centrales de la representación son elementos con fuerte frecuencia de aparición y un puesto de importancia medio bajo. Pues, para ello, conviene calcular la media de las frecuencias de los elementos de representación y la media de los puestos medios.

Los elementos del núcleo central son los que cumplen con la doble exigencia siguiente:

- Frecuencia> frecuencia media

- Puesto medio< media de los puestos medios

El cuadro 2 nos permite clasificar así los términos del campo representacional de nuestras poblaciones en 4 zonas distintas explicitadas en la parte «metodología»: el núcleo central, la primera periferia, la zona de los elementos contrastados y la segunda periferia.

Esta técnica nos permitirá valorar la existencia o no de diferencias entre las representaciones de las personas participantes en la experiencia. Con la ayuda del indicio de Jaccard trataremos de entender en qué consisten dichas diferencias de representaciones, y en particular qué dimensiones las estructuran.

\section{LA ESTRUCTURA DE LA REPRESENTACIÓN}

El objetivo de este análisis va a consistir en valorar la fuerza del vínculo entre las unidades de sentido de la representación. Para ello hemos utilizado pues el indicio de Jaccard. Este indicio valora, para cada par de unidades, con qué frecuencia una está presente cuando una de las dos por lo menos está presente. La matriz de asociaciones obtenida de este modo se verá simplificada por el análisis en Grafo de Extensión Mínima (Minimum Spanning Tree Analysis). Este permite elaborar un grafo no orientado de las asociaciones interunidades.

Esto nos dará una imagen muy sintética de las asociaciones semanticas más fuertes. El grafo obtenido será codificado bajo la forma de una matriz simétrica, en la cual cada unidad aparecerá a la vez en línea y en columna, y en la cual se codificará con unos 1 y unos 0 la presencia o la ausencia de un vínculo entre dos unidades. Para tener una representación gráfica de la red asi obtenida, se utilizará un analisis de correspondencias en la matriz de grafo. Se llama " análisis de la contigüidad» esta aplicación particular del análisis de correspondencias a las matrices de grafo.

\section{RESULTADOS}

\section{LAS REPRESENTACIONES SOCIALES DE LOS GRUPOS DE ESTUDIO A PROPÓSITO DE LA NOCIÓN DE «DERECHO»}

Como aparece anteriormente el método clasificación/frecuencia (Verges 1972; Abric 2003) se impuso con más evidencia para llevar a cabo el trato de los resultados que permitían evidenciar la diversidad de las Representaciones.

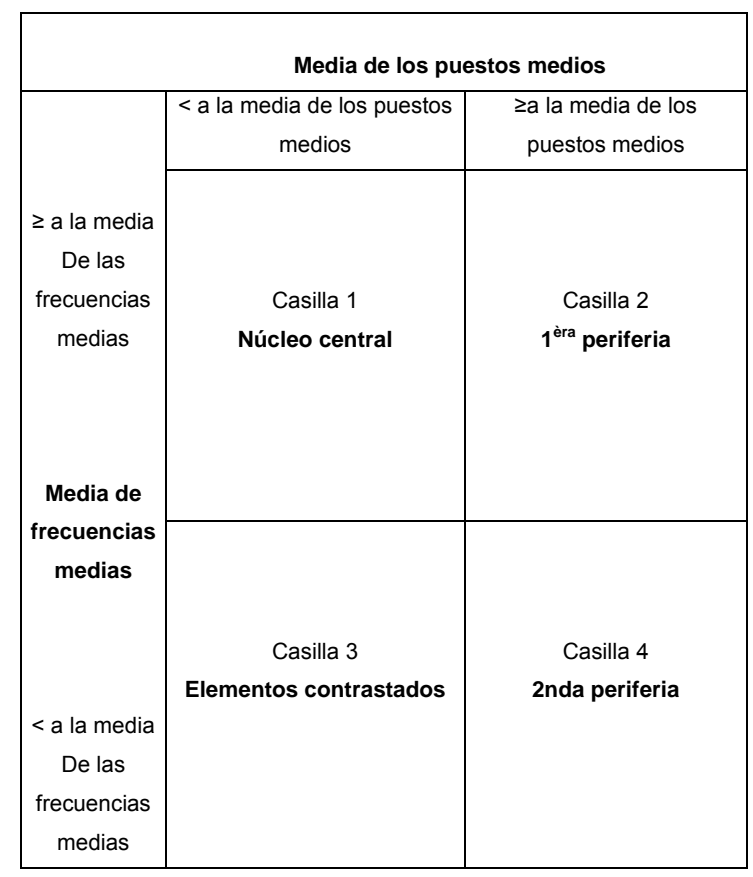

Cuadro 2. Construcción de la representación 


\section{LOS ALUMNOS}

El cuadro 3 permite descubrir los distintos elementos del campo representacional de los alumnos de tercero de BUP:

El núcleo central del campo representacional está compuesto de los términos siguientes: Justicia, derecho del hombre, libertad.

Los elementos periféricos del campo representacional pueden clasificarse de la manera siguiente:

- 1era periferia: Abogado, juzgado, policía, juez.

-Zona de los elementos contrastados: derecho de expresión, derecho del niño, igualdad, leyes, ley, CPE, reglas, derechos de la mujer.

- 2nda periferia: derecho de voto, respeto, cárcel, político.

\begin{tabular}{|c|c|c|}
\hline \multicolumn{3}{|c|}{ MPM 4,49 } \\
\hline \multirow{4}{*}{$=$ MFM } & $<$ MPM & $=$ MPM \\
\hline & 64,3\% Justicia (rm 3,29) & $35,7 \%$ Abogado ( $r m 5,09$ ) \\
\hline & $34,2 \%$ Derechs del & $34,2 \%$ Juzgado (rm 5,57) \\
\hline & hombre(rm3,66) & $31,6 \%$ Juez (rm 5,21) \\
\hline \multirow{5}{*}{$\begin{array}{c}\text { MFM } \\
24,9 \%\end{array}$} & $27,6 \%$ Libertad $(\mathrm{rm} \mathrm{3,13)}$ & 30,1\% Policía (rm 6,42) \\
\hline & & \\
\hline & 23,0\% Derecho de Expresi & \\
\hline & $(\mathrm{rm} 4,40)$ & \\
\hline & $21,4 \%$ Derechodel niño & $24,5 \%$ Derecto de voto \\
\hline \multirow[t]{9}{*}{$<$ MFM } & $(\mathrm{rm} \mathrm{4,05)}$ & $(\mathrm{rm} \mathrm{5,25)}$ \\
\hline & $20,9 \%$ Igualdad (rm 3,59) & 14,3\% Cárcel (rm 8,75) \\
\hline & $20,4 \%$ Leyes (rm 3,88) & 16,3\% Política (rm 4,61) \\
\hline & $19,9 \%$ Respeto ( $r m 3,97$ ) & \\
\hline & $16,8 \%$ Ley $(\mathrm{rm} \mathrm{2,76)}$ & \\
\hline & 14,3\% CPE (rm 4,39) & \\
\hline & $12,2 \%$ Reglas (rm 3,46) & \\
\hline & $11,7 \%$ Derechode lamujer & \\
\hline & $(\mathrm{rm} 4,00)$ & \\
\hline
\end{tabular}

Cuadro 3- Frecuencia y puesto medio de las palabras citadas por los alumnos de 3ro

\section{LOS DOCENTES}

Del mismo modo que en el cuadro anterior, el cuadro 4 permite descubrir los distintos elementos del campo representacional de los docentes:

El núcleo central del campo representacional está compuesto de los términos siguientes: justicia, respeto, deber, derechos del hombre, deberes, ley, leyes.

Los elementos periféricos del campo repre- sentacional pueden clasificarse de la manera siguiente:

- 1era periferia: libertad, abogado, juez, código penal.

-Zona de los elementos contrastados: reglas, derechos y deberes, igualdad, derecho del niño, democracia, derecho de la mujer.

- 2nda periferia: juzgado, prohibiciones, código civil, permiso, estudios de derecho, derecho laboral, sanciones, sociedad, tener el derecho.

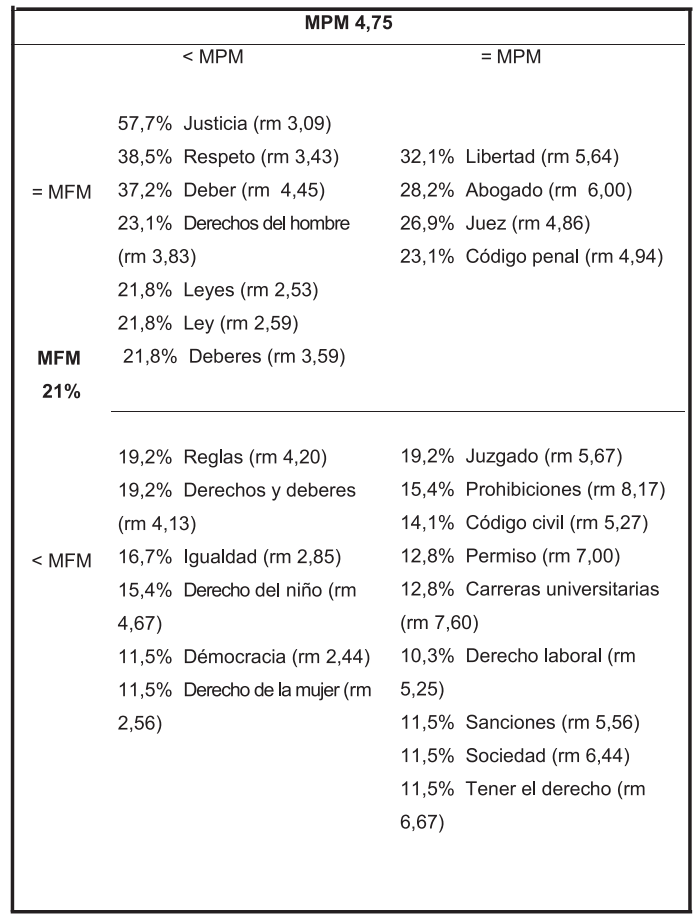

Cuadro 4- Frecuencia y puesto medio de las palabras citadas por los docentes

\section{LOS JURISTAS}

Del mismo modo que en el cuadro anterior, el cuadro 5 permite descubrir los distintos elementos del campo representacional de los juristas:

El núcleo central del campo representacional está compuesto de los términos siguientes: justicia, abogado, ley, juzgado.

Los elementos periféricos del campo representacional pueden clasificarse de la manera siguiente:

- 1era periferia: sociedad.

-Zona de los elementos contrastados: juristas, reglas, libertad, equidad, defensa, igualdad. 
-2nda periferia: juez, magistrado, leyes, código, jurisprudencia, contrato, estudios de derecho.

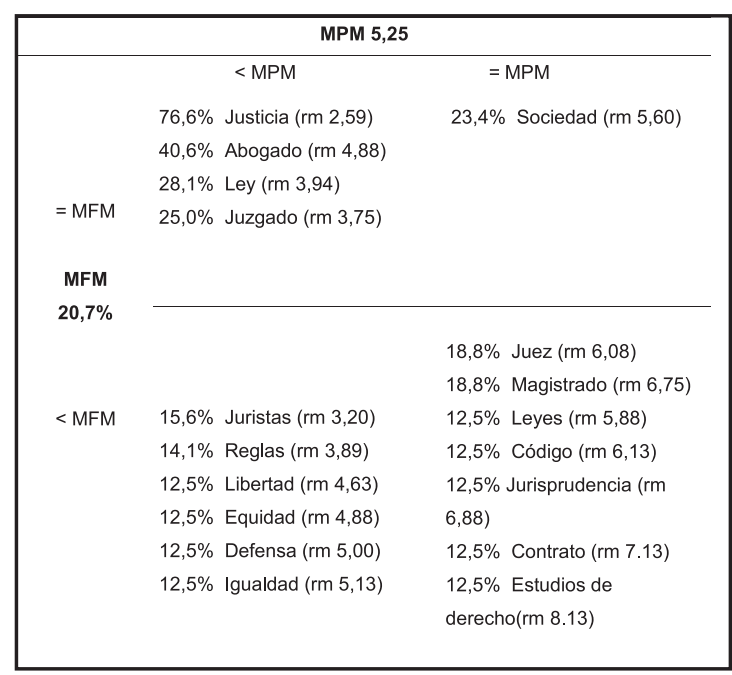

Cuadro 5- Frecuencia y puesto medio de las palabras citadas por los «juristas»

\section{ESTRUCTURA DE LAS REPRESENTACIONES}

Como lo hemos evocado ya, hemos identificado la estructura de las representaciones mediante el indicio de Jaccard y la hemos representado mediante un Grafo de Extensión Mínimo (Minimum Spanning Tree Analysis) que permite elaborar un grafo no orientado de las asociaciones. Gracias a este método, cada persona se ve proyectada según la media de puestos de las palabras que ha utilizado.

Al representar los estatutos de las personas (Docentes, Alumnos, Peritos) por símbolos gráficos, resulta posible observar puntos de vista diferentes sobre un mismo espacio semántico (no métrico).

No existe ningún cuestionario- test estadístico asociado a este procedimiento ya que la representación por análisis de contigüidad es pura comodidad que garantiza que las aristas del grafo no se superponen y no tiene sentido métrico.

No hay pues modelo de distribución natural para estos objetos. El análisis pretende dar una visualización inmediata y en dos dimensiones de una estructura semántica compleja.

Por analogía con la interpretación de los factores en los análisis dimensionales, indicaremos en los gráficos interpretaciones de subregiones de la figura, en función de las unidades que se encuentren reunidas.

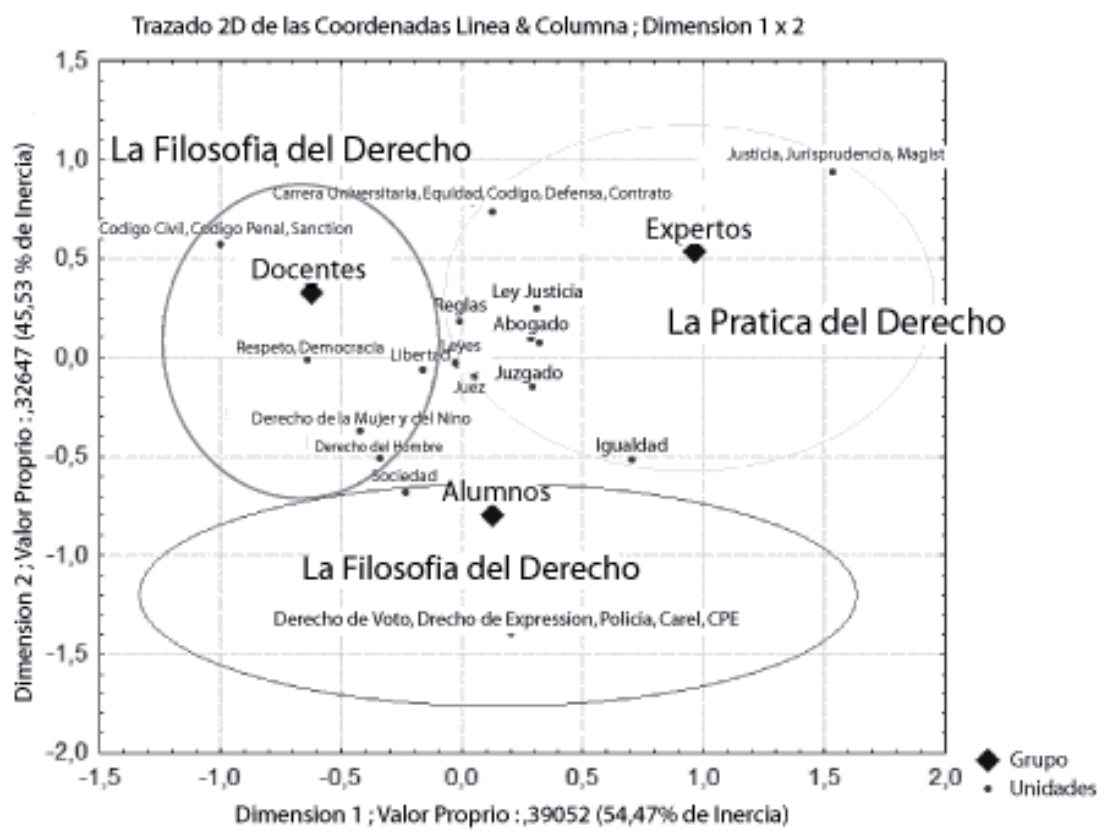

Figura - Naturaleza de la estructura de las représentaciones de las diferentes poblaciones observadas 


\section{DISCUSIÓN}

Como podemos constatarlo, el número y el tipo de ítem recogidos por el método de asociación de palabras (es decir el tamaño y la naturaleza del campo representacional) difieren para cada grupo. ¿Cómo puede explicarse? El campo representacional más amplio es el de los docentes de colegio.

Varios factores pueden explicarlo tales como la diversidad de las asignaturas enseñadas por los docentes de nuestra muestra (remitiendo a carreras escolares fuertemente diversificadas); o bien porque el hecho de enseñar necesita conocer numerosos objetos sociales en su globalidad para adaptarse mejor a un público heteróclito y de nivel variable $\left(6^{\circ}-3^{\circ}\right)$.

El campo representacional más restringido es el de los juristas. De hecho, parece que la peritación en el sector permite elaborar una representación fuertemente consensual del objeto (vinculada a la cultura jurídica, basada en la adquisición de nociones y valores comunes) y por lo tanto más operacional.

El campo representacional de los alumnos es también poco amplio. Como Tapp y Lévine (1977), podemos considerar que éste no es definitivo vista la edad de la muestra del estudio. En realidad, se puede considerar que su representación del objeto "derecho» resulta todavía incompleta y/o fuertemente normativa.

Además, la similitud de campo representacional intergrupo es bastante débil en la medida que un poco más del 6/10 de los ítems no son comunes a nuestras tres poblaciones. Esto confirma que "el derecho» es un objeto social no consensual, a propósito del cual existe discrepancia de puntos de vista según las categorias sociales.

Así, el núcleo central de la muestra de alumnos parece constituido de los términos siguientes: «justicia», «derecho del hombre» y «libertad».

El núcleo central de los docentes, el más amplio de los tres, parece constituido de los términos siguientes: «justicia», «respeto», «deber», «derecho del hombre», «leyes», «ley»y «deberes».

Por fin, el núcleo central de los peritos parece constituido por los términos siguientes: «justicia», «abogado», «ley»y «juzgado».
Podemos notarlo, el término «justicia» aparece dentro del núcleo central de cada una de las tres poblaciones. El término «derecho del hombre» aparece en el núcleo central de las poblaciones de alumnos y de los docentes y el término «ley» aparece en el núcleo central de las poblaciones de docentes y peritos. En vista de nuestros datos teóricos, estas primeras observaciones permiten hacer dos constataciones: primero, en lo que a los adolescentes se refiere, comprobamos como lo señalaban ya los trabajos de Kourilsky (1991 ,2002) que asocian el derecho a la «libertad». En vista de los datos teóricos del derecho, una cuestión queda sin respuesta en lo que se refiere al término «justicia» recurrente en nuestras tres poblaciones. Detrás del uso de este mismo término ¿habrá la misma visión de la justicia? Porque, según nuestros datos teóricos, la pregunta que se nos ocurre enseguida es: ¿Estamos en una visión idealista o en una visión positivista?

El análisis mediante el índice de Jaccard puede ayudamos a entender en qué aspectos difieren los campos representacionales de nuestras poblaciones.

En efecto el de los docentes parece articularse más en tomo a los grandes temas que son la libertad, la democracia, el respeto, los derechos del hombre en general y la referencia a los códigos (civil y penal) fruto de la revolución francesa. El campo representacional de los peritos se expresa más en tomo a la práctica del derecho.

Se encuentran en particular actores y lugares tales como los magistrados, jueces, abogados y juzgado. Se halla también la dimension práctica del derecho, con la referencia al contrato, a la regla y a la jurisprudencia. Por fin, decubrimos también la referencia a diversos principios tales como la defensa, la igualdad y la equidad.

En cuanto a los alumnos, tienen ellos un campo representacional muy distinto y que se arraiga más en la realidad cotidiana con conocimientos concretos y palpables. Así, hacen referencia a la policía, a la cárcel y a datos muy relacionadas con un contexto dado: el CPE. Evocan también dos principios, el del derecho de voto y del derecho de expresión.

Estas diferentes observaciones nos llevan a comprobar que aunque ciertos términos sean comunes al nivel de los núcleos centrales, el 
estudio de los campos representacionales muestra que la visión global del objeto permanece distinta. La representación de los docentes se refiere a ciertos grandes principios que han fundado la república, tiende a acercarse a la visión idealista dei derecho. La representación del jurista se arraiga en una realidad que se articula en torno a nociones más concretas propias al ejercicio de su profesión.

Esto puede interpretarse como relativo a una visión más positivista que pretende ser más eficaz.

En cuanto a los adolescentes, parece que su representación se encuentre más arraigada en "una realidad factual de proximidad» que podria pues evolucionar con la experiencia. Es lo que ya nos enseñaban los trabajos de investigadores en psicología en particular Piaget (1932), Percheron (1991).

Estas diferentes observaciones llevan a pensar que si el derecho, como objeto social, está percibido de distintas maneras por grupos sociales que suelen encontrarse en el marco de transmisiones (docentes-adolescentes), de sanciones (juristas-adolescentes) o en un marco cientifico (juristas-docentes), es necesario que sea objeto de mediaciones para que la comprensión y la comunicación entre estos grupos sean realmentes eficaces.

Otra pregunta se plantea también: ¿Tienen los adolescentes que evolucionar? ¿Y hacia qué?, ¿La fase 6 que describe Kohlberg (1971) y que se acerca a la visión idealista del derecho puede constituir un término hacia el cual es preciso que tiendan ellos?

Si como Lehalle y al. (2004) pensamos que el nivel de educación podría tener una influencia en su emergencia, se trata entonces de una verdadera cuestión de sociedad y de un verdadero problema de formación.

\section{CONCLUSIONES}

El estudio realizado tenía sobre todo un carácter descriptivo y exploratorio. Permite no obstante comprobar entre un número no desdeñable de personas que las poblaciones estudiadas es decir alumnos de 3ro, docentes de colegio y juristas no tienen la misma representación del derecho. Unos se hallan en lo vivido, los otros en la filosofía y los últimos en la práctica a menudo abstracta.

Ahora bien, se da por cierto que los individuos buscan cierta coherencia entre lo que piensan y lo que hacen. Los trabajos sobre la disonancia cognitiva (Festinger, 1957,1959) han mostrado por otra parte que el individuo, en una situación dada, intenta actuar de conformidad con lo que opina.

Las representaciones sociales, coma lo sugiere Moscovici ( 1976) constituyen pues una guía para la acción. En efecto, a próposito de un objeto social dado, ellas van a orientar de manera significativa las prácticas de los individuos y de los grupos para con éste.

El sistema central de la representación, al canalizar de modo no negociable las prácticas de un grupo dado, va a organizar y acondicionar las prácticas empleadas para con un objeto social. Por otra parte, para el observador cándido, las prácticas de un individuo exterior a un grupo pueden parecer extrafias o contradictorias, cuando éste trata de integrarse en él.

Pueden explicarse estos comportamientos inadecuados por las diferencias de representaciones del grupo o dei individuo.

Es exactamente lo que, en materia de justicia social en el seno de la escuela, puede explicar los desfases entre los juicios de los alumnos y los de los docentes y provocar incompresiones mutuas.

¿Qué ocurre pues cuando la justicia oficial frecuentemente desencamada ya que con demasiadas referencias a los textos, desea ajustar los comportamientos?

\section{REFERENCIAS}

Abric, J.C. (1976). Jeux, conflits et représentations sociales, Thèse de Doctorat ès Lettres, Universités de Provence.

Abric, J.C. (1994). Pratiques sociales et représentation, Paris: PUF.

Abric, C. (2003). Méthodes d'étude des représentations sociales, Ramonville-Saint-Agne : ERES

Buffelan-Lanore, Y. \&Larribau-Tereyre, V (2007). Droit civil. Première année introduction, Paris : SIREY, 3 - 9

Carbonnier, J. (1991). Droit civil introduction, Paris: Presses Universitaires de France, p81

Colby,A., \& Kohlberg, L. (1987). The measurement of moral judgment ( $\mathrm{Vol}$ 1) Cambridge UK: Cambridge University Press. 
Colby,A., \& Kohlberg, L., Speicher, B., Hewer, A., Candee, D., Gibbs, J.C., \& Power, C. (1987). The measurement of moral judgment ( Vol 2). Cambridge UK: Cambridge University Press.

Courbe,P.(2003).Introductiongénéraleaudroit.Paris:Dalloz

Dabin, J. (1969). Théorie générale du droit. Paris : Dalloz.

Doise, W., Palmonari, A. (2002). L'étude des représentations sociales, Lausanne: Delachaux Niestle

Festinger,L. (1957). A theory of cognitive dissonance, Evanston : Row, Peterson

Festinger, L., Carlsmith, J. M. (1959). Cognitive consequences of forced compliance, Journal of Abnormal and Social Psychology, 58, 203-210

Flament, C. (1962). L'analyse de similitude, Cahiers du Centre de Recherche opérationnelle, 4, 63-97.

Gangloff, B. \& Hardy-Massard, S (2006). Critères Attributifs de Sanction et «Culture de Métier» Approche Comparative dans la Justice Naïve Versus «Experte» Revista Interamericana de Psicologia/Interamerican Journal of Psychology - 2006, Vol. 40 -1, 21-36

Guimelli, C., Rouquette, M.-L. (1992). Contribution du modèle associatif des schèmes cognitifs de base à l'analyse structurale des représentations sociales, $\mathrm{Bu}$ lletin de Psychologie, XLV, 405, 196-202

Kohlberg, L. (1971). From is to ought: How to commit the naturalistic fallacy and get away with the study of moral development, in T.H. Mischel, Cognitive Development and Epistemology, New-York: Academic Press, 151-235.

Kourilsky, C. (1991). Socialisation juridique et identité du sujet, Droit et société, 19-1991, 273-291

Kourilsky, C. (2002). Le citoyen russe aujourd'hui : appartenir à la Russie et regarder l'Europe, Droit et culture, 43,2002/1, 177-204

Lehalle, H., Aris, C., Buelga, S \& Musitu, G. (2004). Développement socio-cognitif et jugement moral. De Kohlberg à la recherche des déterminants de la différenciation du développement moral. L'Orientation Scolaire et Professionnelle, 33(2), 289-314

Moliner, P. (1993). Cinq questions à propos des représentations sociales, Les Cahiers Internationaux de Psychologie Sociale, 20, 5-14.

Moscovici, S. (1961/1976). La psychanalyse, son image et son public, Paris : PUF
Moscovici, S .(1984). Psychologie sociale, Paris : PUF

Pagoni-Andréani, M. (1999). Le développement sociomoral, des théories à l'éducation civique, Villeneuve d'asq: Presses Universitaires du Septentrion.

Percheron, A. (1991). Représentations de la loi et de la justice chez les français de 16 à 21 ans, Droit et société, 19-1991, 385-397

Piaget, J. (1932). Le jugement moral chez l'enfant, Paris : Presses Universitaires de France

Planiol, M. (1934). Traité pratique de droit civil, Paris : Dalloz Rest, J.R. (1979). Revised manual for the Defining Issues Test.Anobjectivetestmoraljudgmentdevelopment. Minneapolis, U.S.A.: Minnesota Moral Research Projects.

Rest, J.R., Davison, M.L., \& Robbins, S. (1978). Age trends in judging moral issues: A review of cross- sectional, longitudinal, and sequential studies of the Defining Issues Test, Child Development, 49, 263-279

Rest J.R., Narvaez, D., Bebeau, M.J., \& Thoma, S.J. (1999). Postconventional moral thinking. A neo-kohlbergiean approach. Mahwah NJ, USA: Lawrence Erlbaum

Sampson, E.E (1977). Psychologie and the American ideal, Journal of Personality and social Psychology, 35, 11, 767-782

Shweder, R.A. (1982b). Liberalism as a destiny: A review of Lawrence Kohlberg, Contemporary Psychology, 27, 6, 421-424

Simpson, E.L.(1974). Moral developmentresearch:Acase of scientific cultural bias, Human Development, 17, 81-106

Snarey, J.R. (1985). Cross-cultural universality of social moral development: A critical review of kohhlbergian research, Psychological Bulletin, 97, 2, p 202-232

Sullivan, E.V. (1977). A study of Kohlberg's structural theory of moral development: A critique of liberal social science ideology, Human Development, 20, 352-376

Tapp, J.L. \& Kohlberg, L. (1971). Developing senses of law and legal justice, Journal of social issues, 27, 2, 65-92

Tapp, J.L. \& Levine F.J.(1977). Law, justice, and the individual in society: Psychological and legal issues, New York, Holt, Rinehart.

Tostain, M. (1999). Psychologie, morale et culture, l'évolution de la morale de l'enfance à l'âge adulte, Grenoble : Presses universitaires de Grenoble

Verges, P. (1992). L'évocation de l'argent : une méthode pour la définition du noyau central d'une représentation, Bulletin de psychologie, XLV, 405, 203-209 